\title{
A Systematic Approach to the Construction of Non-empty Choice Sets*
}

\author{
John Duggan \\ Department of Political Science \\ and Department of Economics \\ University of Rochester
}

May 17, 2004

\begin{abstract}
Suppose a strict preference relation fails to possess maximal elements, so that a choice is not clearly defined. I propose to delete particular instances of strict preferences until the resulting relation satisfies one of a number of known regularity properties (transitivity, acyclicity, or negative transitivity), and to unify the choices generated by different orders of deletion. Removal of strict preferences until the subrelation is transitive yields a new solution with close connections to the "uncovered set" from the political science literature and the literature on tournaments. Weakening transitivity to acyclicity yields a new solution nested between the strong and weak top cycle sets. When the original preference relation admits no indifferences, this solution coincides with the familiar top cycle set. The set of alternatives generated by the restriction of negative transitivity is equivalent to the weak top cycle set.
\end{abstract}

\footnotetext{
${ }^{*}$ I am indebted to Mark Fey and Tasos Kalandrakis for helpful discussions during the
} construction of this paper. 


\section{Introduction}

Consider a strict preference relation over a set of alternatives. If this relation possesses maximal elements, then some alternatives are at least as good as all others, and these are obvious choices. In many applications, however, maximal elements may fail to exist: this problem is common in the analysis of pairwise majority voting, in the choice of a winning sports team, or in the aggregation of multiple choice criteria. In such situations, there are no unambiguous choices. I examine procedures for the construction of non-empty choice sets that are based on the systematic removal of particular instances of strict preferences. The idea is to delete strict preferences until the resulting relation satisfies one of a number of common transitivity properties: transitivity, acyclicity, and negative transitivity. Since particular orders of deletion may lead to different sets of maximal elements, I propose to unify the choices resulting from all possible orders of deletion. As a result of the analysis, I introduce two new solutions concepts, and I provide a new choice-theoretic foundation for the well-known top cycle set.

I first consider removing strict preferences until the resulting subrelation is transitive, stopping at that point. This produces a maximal transitive subrelation of the original preference relation. When the set of alternatives is finite, this subrelation will admit maximal elements, where the original did not. Even in the infinite setting, transitivity will facilitate the search for maximal elements. Since different orders of deletion can produce different choice sets, I take the union of all maximal sets of all maximal transitive subrelations. This procedure for the construction of choice sets has a simple binary representation: the resulting choice set can itself be written as the maximal elements of a new "extended covering" relation, which strengthens the notion of covering from the political science literature and the literature on tournaments. ${ }^{1}$ The choice set generated in this manner, the "extended uncovered set," is therefore a superset of the usual uncovered set. Banks, Duggan, and Le Breton $(2002,2003)$ prove that the uncovered set is nonempty if the set of alternatives is compact and the original strict preference relation is open. Thus, the above procedure succeeds in generating nonempty choice sets quite generally.

While transitivity is conducive to the existence of maximal elements, it is not a necessary condition. A weaker condition is acyclicity - this is still sufficient for existence of maximal elements when the set of alternatives is

\footnotetext{
${ }^{1}$ See McKelvey (1986) and Moulin (1986).
} 
finite, and it is actually necessary for existence of maximal elements in all subsets of alternatives. Thus, I consider modifying the above procedure to remove particular instances of strict preferences until the resulting subrelation is acyclic, rather than waiting until it is fully transitive. Again, different orders of deletion can produce different choice sets, so I take the union of all maximal sets of all maximal acyclic subrelations.

This solution to the problem of constructing choice sets has a novel binary representation, in terms of a relation I call the "trapping" relation. We say an alternative $x$ "traps" $y$ if it is strictly preferred to $y$ and it cannot be reached from $y$ through any finite sequence of strict preferences, and the maximal elements of this relation are the "untrapped set." I show that it coincides with the maximal elements of all maximal acyclic subrelations, and that the untrapped set is nested between the strong and weak top cycle sets. In the absence of indifferences, as in the literature on tournaments, the strong and weak top cycles coincide, and they therefore coincide with the untrapped set. This equivalence yields a previously unknown foundation for the top cycle set in terms of choice from maximal acyclic subrelations. I prove that the strong top cycle set is non-empty under the usual compactness and continuity conditions, from which it follows that the untrapped set is non-empty quite generally.

I also consider the possibility of removing instances of strict preferences until the remaining strict preference relation is not only transitive, but negatively transitive. These are the subrelations that correspond to complete rankings of alternatives. The set of alternatives that appears at the top of each such maximal "sub-ranking" coincides with the weak top cycle. We conclude that, in the absence of indifferences, the choices generated by the maximal acyclic subrelations and by the maximal negatively transitive subrelations are equivalent.

\section{Framework}

I will make use of the following conceptual apparatus for an arbitrary binary relation, say $B$, on a set $X$. While $B$ consists of ordered pairs of elements of $X$, it is common to write $x B y$ for $(x, y) \in B$. For $x \in X$, let

$$
\begin{aligned}
B(x) & =\{y \in X \mid y B x\} \\
B^{-1}(x) & =\{y \in X \mid x B y\}
\end{aligned}
$$


denote the upper and lower sections of $B$ at $x$. Given a subset $Y \subseteq X$, say $B$ is a relation on $Y$ if $B \subseteq Y \times Y$. Let $\mathcal{P}(B)$ denote the asymmetric part of $B$, i.e., for all $x, y \in X, x \mathcal{P}(B) y$ if and only if $x B y$ and not $y B x$. Let $\mathcal{T}(B)$ denote the transitive closure of $B$, i.e., for all $x, y \in X, x \mathcal{T}(B) y$ if and only if there exist a natural number $n$ and elements $x_{1}, \ldots, x_{n} \in X$ such that

$$
x=x_{1} B x_{2} \cdots x_{n-1} B x_{n}=y .
$$

Thus, $x \mathcal{T}(B) y$ means that we may move from $x$ to $y$ in a finite number of "B-steps." Duggan (1999) gives a characterization of the negation of $\mathcal{T}(B)$, showing that $x \mathcal{T}(B) y$ fails to hold if and only if $y$ "traps" $x$, in a sense: there is a partition $\left\{X_{1}, X_{2}\right\}$ of $X$ such that, for all $z \in X_{1} \cup\{x\}$ and all $w \in X_{2} \cup\{y\}$, not $z B w$. That is, there is no way to pass via $B$ from a certain set around $x$ to the remaining set around $y$.

As usual, $B$ is transitive if, for all $x, y, z \in X, x B$ y $B z$ implies $x B z$. A subset $Y \subseteq X$ is a $B$-cycle if, for all $x, y \in Y$, we have $x \mathcal{T}(B) y$ and $y \mathcal{T}(B) x$. We say $B$ is acyclic if there does not exist a $B$-cycle, i.e., there do not exist a natural number $n$ and elements $x_{1}, \ldots, x_{n} \in X$ such that

$$
x_{1} B x_{2} \cdots x_{n-1} B x_{n} B x_{1} .
$$

We say $B$ is negatively transitive if, for all $x, y, z \in X, x B y$ implies either $x B z$ or $z B y$. Let $M(B \mid Y)$ denote the elements of $Y$ that are $B$-maximal in $Y$, i.e.,

$$
M(B \mid Y)=\{x \in Y \mid \text { for all } y \in Y, y B x \text { implies } x B y\} .
$$

When $Y=X$, we write $M(B)$ for this set and refer to the elements simply as " $B$-maximal." When $Y$ is finite, transitivity, acyclicity, and negative transitivity of $B$ are all sufficient for the existence of an element that is $B$-maximal in $Y$.

Now let $P$ denote an asymmetric relation on a set $X$, and let $R$ denote the complete relation such that, for all $x, y \in X, x P y$ if and only if not $y R x$. We interpret elements of $X$ as alternatives, $P$ as a strict preference relation, and $R$ as the associated weak preference. Let $I$ denote the associated indifference relation, so that $x I y$ holds if and only if neither $x P y$ nor $y P x$. Say $P$ is total if, for all distinct $x, y \in X$, either $x P$ or $y P x$. This condition formalizes the idea that no two distinct alternatives are indifferent according to $P$. Because $P$ is asymmetric,

$$
M(P)=\{x \in X \mid \text { there is no } y \in X \text { such that } y P x\},
$$


so an alternative is $P$-maximal if and only if there is no alternative strictly preferred to it. Because $R$ is complete,

$$
M(R)=\{x \in X \mid \text { for all } y \in X, \text { we have } x R y\},
$$

so an alternative is $R$-maximal if and only if it is weakly preferred to all others. Of course, $M(P)=M(R)$. In the sequel, we take the relations $P$ and $R$ as fixed.

Negative transitivity of $P$ is equivalent to transitivity of $R$, which means that the set of alternatives can be ranked by these preference relations. Clearly, negative transitivity of $P$ implies transitivity, which implies acyclicity. All three conditions are sufficient for existence of $P$-maximal elements in finite sets, and acyclicity of $P$ is actually necessary for existence of maximal elements in all finite subsets of $X$, so it cannot be relaxed much while maintaining the possibility of $P$-maximal elements. Dropping finiteness of $X$, it is known that acyclicity is still sufficient for existence of $P$-maximal elements quite generally, and it can be weakened even further. Assuming $X$ is compact and $P^{-1}(x)=\{y \in X \mid x P y\}$ is open for all $x \in X$, there exists a $P$-maximal element if and only if the following condition holds: for every finite set $Y \subseteq X$, there exists $x \in X$ such that, for all $y \in Y, x R y{ }^{2}$

In some applications, however, acyclicity of $P$ turns out to be restrictive: when $P$ represents the preferences of a society through a given aggregation mechanism, for example, it is common for $P$ to violate this regularity condition. The well-known Condorcet Paradox demonstrates this for social preferences derived from majority rule: if three voters have rankings, $x y z$, $y z x, z x y$, respectively, over three alternatives, and if $P$ represents the strict majority preference relation, then we have $x P$ y $P z P x$, a cycle, so that $M(P)=\emptyset$. The literature in social choice theory has generalized this example, establishing that social preference cycles are unavoidable when decision-making authority is decentralized. ${ }^{3}$ To address the absence of maximal elements, several methods for constructing non-empty choice sets have been considered.

One of the primary alternatives to maximality is the "top cycle." Following Schwartz (1986), we define two versions of the top cycle, one a subset of the other. ${ }^{4}$ The weak top cycle set, denoted $W T C$, is defined as the maximal

\footnotetext{
${ }^{2}$ See Walker (1977) and Austen-Smith and Banks (1999).

${ }^{3}$ See Sen (1970), Mas-Colell and Sonnenschein (1972), Schwartz (1986, 2001), and Banks (1995).

${ }^{4}$ Schwartz (1986) refers to these sets as GETCHA and GOCHA.
} 
elements of the transitive closure of $R$, i.e.,

$$
W T C=M(\mathcal{T}(R)) .
$$

In other words, an alternative $x$ is in the weak top cycle if and only if, for all $y \in X, x \mathcal{T}(R) y$. Clearly, $\mathcal{T}(R)$ is transitive, so that the weak top cycle is non-empty as long as $X$ is finite. More generally, if $X$ is a compact topological space and $P^{-1}(x)$ is open for all $x \in X$, then the weak top cycle is non-empty.

The strong top cycle set, denoted $S T C$, is defined as the maximal elements of the transitive closure of $P$, i.e.,

$$
S T C=M(\mathcal{T}(P)) .
$$

In other words, $x$ is in the strong top cycle if and only if, for all $y \in X$, $y \mathcal{T}(P) x$ implies $x \mathcal{T}(P) y$. An alternative characterization of the strong top cycle is as follows. A subset $Y \subseteq X$ is undominated if, for all $x \in Y$ and all $y \in X \backslash Y$, we have $x R y$. Letting $\mathbf{Y}$ denote the collection of undominated $P$-cycles, we have

$$
S T C=\bigcup \mathbf{Y}
$$

It is well-known that $S T C \subseteq W T C$ generally. Furthermore, if $P$ is total, then the two sets coincide. In this case, I refer simply to the "top cycle set" and use the notation $T C$.

As above, $\mathcal{T}(P)$ is transitive, and finiteness of $X$ implies the strong top cycle is non-empty. More general conditions for non-emptiness of the strong top cycle are not immediate, as the relation $\mathcal{T}(P)$ does not generally possess desirable continuity properties. The next result establishes general conditions for non-emptiness of the strong top cycle and implies the claimed result for the weak top cycle as well.

Proposition 1 Assume $X$ is compact and $P^{-1}(x)$ is open for all $x \in X$. Then $S T C \neq \emptyset$.

With the continuity assumption of the proposition, a necessary and sufficient condition for the existence of $P$-maximal elements is that for every finite subset $Z \subseteq X$, there exists $x \in X$ such that, for all $y \in Z$, we have $x R y$. Under this condition, $M(P) \neq \emptyset$, and then non-emptiness of the 
strong top cycle follows from $M(P) \subseteq S T C$. Thus, to prove the proposition, assume there is a finite set $Z \subseteq X$ such that, for all $x \in X$, there exists $y \in Z$ with $y P x$. Since $\mathcal{T}(P)$ is transitive, there exists $z^{*} \in M(\mathcal{T}(P) \mid Z)$. Then $Z^{*}=\mathcal{T}(P)\left(z^{*}\right)$ is non-empty, since there exists $y \in Z$ with y $P z^{*}$, and I claim that $Z^{*}$ is an undominated $P$-cycle. Clearly, for all $x \in Z^{*}$ and all $y \notin Z^{*}$, we have $x R y$, so $Z^{*}$ is undominated. Now take any $x \in Z^{*}$, so $x \mathcal{T}(P) z^{*}$. If $x \in Z$, then $z^{*} \mathcal{T}(P) x$ follows by maximality of $z^{*}$. If $x \in Z^{*} \backslash Z$, then there exists $y \in Z$ such that $y P x$, so $y \mathcal{T}(P) z^{*}$. Again, by maximality of $z^{*}$, we have $z^{*} \mathcal{T}(P) y$, and then $z^{*} \mathcal{T}(P)$ y $P x$ implies $z^{*} \mathcal{T}(P) x$. To see that $Z^{*}$ is a $P$-cycle, take $x, y \in Z^{*}$. By the preceding argument, we have

$$
x \mathcal{T}(P) z^{*} \mathcal{T}(P) \text { y } \mathcal{T}(P) z^{*} \mathcal{T}(P) x,
$$

so $z^{*}$ is a $P$-cycle. Therefore, $Z^{*}$ is a non-empty, undominated $P$-cycle. This implies $S T C \neq \emptyset$, completing the proof of the proposition.

Following McKelvey (1986), we say $x$ covers $y$, denoted $x C y$, if $x P y$, $P(x) \subseteq P(y)$, and $R(x) \subseteq R(y)$. Note that $C$ is transitive, though it does not typically possess desirable continuity properties. The uncovered set, written $U C$, is the set of $C$-maximal elements: $U C=M(C)$. Alternatively, an alternative $x$ is uncovered if the following "two-step principle" holds: for every $y \in X$, either $x R y$, or there exists $z \in X$ such that $x R z P y$, or there exists $z \in X$ such that $x P z R y$. Because covering is transitive, it immediately follows that the uncovered set is always non-empty when $X$ is finite. The following result, due to Banks, Duggan, and Le Breton (2002, 2003), establishes non-emptiness of the uncovered set under the same general conditions used for the strong top cycle.

Proposition 2 Assume $X$ is compact and $P^{-1}(x)$ is open for all $x \in X$. Then $U C \neq \emptyset$.

It is known that the strong top cycle and the uncovered set are not logically nested. ${ }^{5}$ That the uncovered set is a subset of the weak top cycle set follows directly from the two-step principle. Several different definitions of the covering relation have appeared in the literature: Fishburn (1977) drops the $R(x) \subseteq R(y)$ condition; Miller (1980) drops the $P(x) \subseteq P(y)$ condition; while Duggan (2004) strengthens the definition of covering by

\footnotetext{
${ }^{5}$ See Duggan and Le Breton (1999) for examples showing that either can be a proper subset of the other.
} 
demanding $R(x) \subseteq P(y)$. All of these definitions are equivalent when $P$ is total, precluding indifferences. The analysis of the next section provides a foundation for a strengthening of McKelvey's covering, which can yield larger choice sets.

\section{Choice via Transitivity: The Extended Uncov- ered Set}

We first consider the choices generated by maximally transitive subrelations. Let $\mathbf{P}_{T}$ denote the transitive subrelations of $P$, i.e.,

$$
\mathbf{P}_{T}=\{\hat{P} \subseteq P \mid \hat{P} \text { is transitive }\},
$$

and let $\mathbf{P}_{T}^{*}$ denote the elements of $\mathbf{P}_{T}$ that are maximal with respect to set-inclusion. The main result in this section establishes a simple binary characterization of the choices generated by transitive subrelations, i.e., I show that these choice sets can be viewed as maximal elements of a particular binary relation.

We say $x$ extended covers $y$, written $x X C y$, if $x C y$ and

$$
P(x) \subseteq \bigcap_{z \in P^{-1}(y)} P(z) .
$$

That is, we add to the definition of covering the requirement that for all $s, t \in X$, if $s P x$ and $y P t$, then $s P t$. The extended uncovered set, written $U X C$, is the set of $X C$-maximal elements: $U X C=M(X C)$. It is easily verified that an alternative belongs to the extended uncovered set if and only if it respects the following "three-step principle": for all $y \in X$, at least one of
(i) $\quad x R y$
(ii) there exists $w \in X$ such that $x P w R y$
(iii) there exists $z \in X$ such that $x R z P y$
(iv) there exist $s, t \in X$ such that $x P s R P y$ 
holds. The following result clarifies the relationships between the extended uncovered set and the solutions introduced above. As a consequence, it follows from Proposition 2 that the extended uncovered set is non-empty under the usual general conditions: it is sufficient that $X$ is compact and, for all $x \in X$, the lower section $P^{-1}(x)$ is open.

\section{Theorem 1}

$$
U C \subseteq X U C \subseteq W T C
$$

The first inclusion follows directly from the observation that covering implies extended covering. The second inclusion follows directly from the threestep principle. To see that both inclusions may be strict, consider the following examples. First, let $X=\{a, b, c, d, e\}$, and let $P=\{(a, b),(a, c),(a, d)$, $(b, c),(c, d),(d, e),(e, a),(e, b)\}$; then $b \in U X C \backslash U C$. Second, simply reverse the strict preference $(c, e)$ to $(e, c)$, and we have $b \in T C \backslash U X C$. Since $P$ is total in the latter example, it shows that the extended uncovered set may be a proper subset of the strong top cycle. For the opposite inclusion, let $X=\{a, b, c\}$ and $P=\{(a, b),(b, c)\}$, and note that $S T C=\{a\}$, while $U X C=X$.

Our interest in the extended uncovered set lies in its connection to choice sets generated from transitive subrelations. The next result shows that these connections are tight: an alternative is maximal for a maximal transitive subrelation if and only if it belongs to the extended uncovered set.

\section{Theorem 2}

$$
U X C=\bigcup_{\hat{P} \in \mathbf{P}_{T}^{*}} M(\hat{P})
$$

To prove the theorem, take any $\hat{P} \in \mathbf{P}_{T}^{*}$ and any $y \in M(\hat{P})$. Suppose $y \notin U X C$, so there exists $x \in X$ such that $x X C y$. Define $P^{\prime}=\hat{P} \cup\{(x, y)\}$, and let $\bar{P}$ be the transitive closure of $P^{\prime}$. To see that $\bar{P} \subseteq P$, take any $s, t \in X$ such that $s \bar{P} t$, so there exist a natural number $n$ and alternatives $x_{1}, \ldots, x_{n} \in X$ such that

$$
s=x_{1} P^{\prime} x_{2} \cdots x_{n-1} P^{\prime} x_{n}=t .
$$

Consider the smallest $n$ for which there exist such $x_{1}, \ldots, x_{n}$. If $\left(x_{k}, x_{k+1}\right)=$ $(x, y)$ for no $k=1, \ldots, n$, then, by transitivity of $\hat{P}$, we have $s P^{\prime} t$. If 
$\left(x_{k}, x_{k+1}\right)=(x, y)$ for some $k=1, \ldots, n$, then by choice of $n$ this holds for exactly one $k$. By transitivity of $\hat{P}$, we have $n=2,3$, or 4 , with four cases:

- $s=x X C y=t$

- $s \hat{P} x X C y=t$

- $s=x X C y \hat{P} t$

- $s \hat{P} x X C y \hat{P} t$.

Since $\hat{P} \subseteq P, s P t$ follows from $x X C y$ in all three cases. Therefore, $\bar{P} \subseteq P$, but this contradicts maximality of $\hat{P}$. Therefore, $y \in U X C$.

Now take any $x \in U X C$. Construct the transitive subrelation $P^{\prime}$ of $P$ in three steps. Step 1: For each $y \in X$ such that $x P y$, let $(x, y) \in P^{\prime}$. Step 2: For each $y \in X$ such that (A) y $P x$ and (B) there does not exist $w \in X$ such that $x P w R y$, if there exists $z(y) \in X$ such that $x I z(y) P y$, then let $(z(y), y) \in P^{\prime}$. Step 3: For each $y \in X$ such that (A) y $P x$, (B) there does not exist $w \in X$ such that $x P w R y$, and (C) there does not exist $z(y)$ such that $x I z(y) P y$, if there exist $s(y), t(y) \in X$ such that $t(y) P x$ and $x P s(y) R t(y) P y$, then let $(t(y), y) \in P^{\prime}$. This completes the definition of $P^{\prime}$. Clearly, $P^{\prime} \subseteq P$, and $x$ is $P^{\prime}$-maximal.

I claim that $P^{\prime}$ is transitive, for suppose $a P^{\prime} b P^{\prime} c$. Since $x$ is $P^{\prime}$ maximal, we have $b \neq x$. So there exists $y \in X$ such that $c=y$ and either $b=z(y)$ or $b=t(y)$. In the latter case, by construction we have $b=t(y) P x$, and there exists $s(y)$ such that $x P s(y) R t(y)$. Then, however, we cannot have $a P^{\prime} t(y)=b$ : in steps 2 and 3 , condition (B) is not fulfilled. Therefore, $b=z(y)$, and we have $b I x$. If $x=a$, then we have $x P^{\prime} b$, implying $x P b$, a contradiction. Thus, $x \neq a$, and there exists $y^{\prime} \in X$ such that $b=y^{\prime}$ and either $a=z\left(y^{\prime}\right)$ or $a=t\left(y^{\prime}\right)$. In both cases, we have $b=y^{\prime} P x$, a contradiction. Therefore, $P^{\prime}$ is indeed vacuously transitive.

Now define the class

$$
\hat{\mathbf{P}}=\left\{Q \subseteq P \mid P^{\prime} \subseteq \hat{P} \text { and } Q \text { is transitive }\right\}
$$

of transitive subrelations of $P$ containing $P^{\prime}$. Since $P^{\prime} \in \hat{\mathbf{P}}$, this set is nonempty. Now take any chain $\mathbf{Q} \subseteq \hat{\mathbf{P}}$, and let $Q=\bigcup \mathbf{Q}$. Clearly, $P^{\prime} \subseteq Q \subseteq P$. That $Q$ is transitive follows because the class of transitive relations is upward closed. (See Duggan, 1999.) Therefore, by Zorn's lemma, $\hat{\mathbf{P}}$ contains a relation, say $\hat{P}$, that is maximal in $\hat{\mathbf{P}}$ with respect to set-inclusion. 
Finally, I claim that $x \in M(\hat{P})$, for suppose $y \hat{P} x$. By the three-step principle, at least one of (i), (ii), (iii), or (iv) holds. Since $y \hat{P} x$ implies y $P x$, (i) cannot hold. If (ii) holds, then we have $x P z R y$, and by construction we have $x P^{\prime} z$, implying $x \hat{P} z$. Then from $y \hat{P} x \hat{P} z$ and transitivity of $\hat{P}$, we have y $\hat{P} z$, which implies y $P$, a contradiction. Thus, (ii) does not hold. If (iii) holds, then we have $x I z P y$, and by construction we have $z P^{\prime} y$, implying $z \hat{P} y$. Then from $z \hat{P}$ y $\hat{P} x$ and transitivity of $\hat{P}$, we have $z \hat{P} x$. But this implies $z P x$, a contradiction. Thus, (iii) does not hold, and therefore (iv) obtains. Thus, we have $x P s R P y$. Furthermore, we have t $P x$, for otherwise we have $x R t P y$, which is impossible if (ii) and (iii) are false. Then, by construction, we have $t P^{\prime} y$ and $x P^{\prime} s$, implying $t \hat{P} y$ and $x \hat{P} s$. From $t \hat{P}$ y $\hat{P} x \hat{P} s$ and transitivity of $\hat{P}$, we have $t \hat{P} s$, which implies $t P s$, a final contradiction. Therefore, $x \in M(\hat{P})$. This completes the proof of the theorem.

\section{Choice via Acyclicity: The Untrapped Set}

In the previous section, we considered removing strict preferences until the resulting subrelation is transitive. The condition of acyclicity is weaker than transitivity, however, and potentially allows us to stop before arriving at a fully transitive subrelation. Let

$$
\mathbf{P}_{A}=\{\hat{P} \subseteq P \mid \hat{P} \text { is acyclic }\}
$$

denote the acyclic subrelations of $P$, and let $\mathbf{P}_{A}^{*}$ denote the elements of $\mathbf{P}_{A}$ that are maximal with respect to set-inclusion. The main result of this section gives a simple binary characterization of choices generated by acyclic subrelations.

We say $x$ traps $y$, written $x T y$, if $x P y$ and not $y \mathcal{T}(P) x$. Equivalently, $x$ traps $y$ if it is preferred to $y$ and there exists a partition $\left\{X_{1}, X_{2}\right\}$ of the set of alternatives such that $x \in X_{1}, y \in X_{2}$, and, for all $w \in X_{1}$ and all $z \in X_{2}$, $w R z{ }^{6}$ The trapping relation is not generally transitive, but it is acyclic: if $x_{1} T x_{2} \cdots x_{n} T x_{1}$, then we have $x_{2} \mathcal{T}(P) x_{1}$, contradicting $x_{1} T x_{2}$. The untrapped set, written $U T$, is the set of $T$-maximal elements: $U T=M(T)$. Clearly, alternative $x$ is untrapped if and only if the following implication holds: for all $y \in X, y P x$ implies $x \mathcal{T}(P) y$. Because trapping is acyclic,

\footnotetext{
${ }^{6}$ For one direction, simply set $X_{2}=\mathcal{T}(P)^{-1}(y) \cup\{y\}$ and $X_{1}=X \backslash X_{2}$. The remaining direction is obvious.
} 
it immediately follows that the untrapped set is always non-empty when $X$ is finite. The next result establishes the close relationship between the untrapped set and the top cycle sets. As a consequence, with Proposition 1 , it follows that the untrapped set is non-empty under the usual general conditions: it is sufficient that $X$ is compact and, for all $x \in X$, the lower section $P^{-1}(x)$ is open.

\section{Theorem 3}

$$
S T C \subseteq U T \subseteq W T C
$$

To prove the first inclusion, it suffices to show that $T \subseteq \mathcal{P}(\mathcal{T}(P))$. To this end, suppose $x T y$, so $x P y$ and not $y \mathcal{T}(P) x$. The former condition clearly implies $x \mathcal{T}(P) y$, and then $x \mathcal{P}(\mathcal{T}(P))$ y follows. For the second inclusion, it suffices to show that $\mathcal{P}(\mathcal{T}(R)) \subseteq T$. Suppose $x \mathcal{T}(R)$ y but not $y \mathcal{T}(R) x$. The latter implies not y $R x$, which implies $x P y$, and it also precludes the possibility that $x \mathcal{T}(P) y$. Therefore, $x T y$, as required. This proves the theorem.

That each inclusion in Theorem 3 may be strict follows from two examples. First, let $X=\{a, b, c, d\}$ and $P=\{(a, b),(b, c),(c, d),(d, b)\}$; then $S T C=\{a\}$, while $U T=\{a, c, d\}$. Second, let $X=\{a, b, c\}$ and $P=\{(a, b),(b, c)\}$; then $U T=\{a, c\}$, while $W T C=X$.

Given Theorem 3, the following corollary is immediate: when $P$ is total, the strong and weak top cycle sets coincide, and therefore they both reduce to the untrapped set. Thus, for the case of tournaments, we give a new interpretation of the familiar top cycle and establish its connections to acyclic choice.

Corollary 1 Assume $P$ is total. Then $U T=T C$.

Our interest in the untrapped set lies in its connection to choice sets generated from acyclic subrelations. The next result shows that these connections are tight: an alternative is maximal for a maximal acyclic subrelation if and only if it is untrapped.

\section{Theorem 4}

$$
U T=\bigcup_{\hat{P} \in \mathbf{P}_{A}^{*}} M(\hat{P})
$$


To prove the theorem, take any $\hat{P} \in \mathbf{P}_{A}^{*}$ and any $y \in M(\hat{P})$. Suppose $y \notin U T$, so there exists $x \in X$ such that $x T y$. Define $P^{\prime}=\hat{P} \cup\{(x, y)\}$. Since $x P y$, we have $P^{\prime} \subseteq P$. Moreover, $P^{\prime}$ is acyclic, for suppose there exist $s \in X$ such that $s \mathcal{T}\left(P^{\prime}\right) s$, so there exist a natural number $n$ and alternatives $x_{1}, \ldots, x_{n} \in X$ such that

$$
s=x_{1} P^{\prime} x_{2} \cdots x_{n-1} P^{\prime} x_{n}=s .
$$

Since $\hat{P}$ is acyclic, we must have $\left(x_{k}, x_{k+1}\right)=(x, y)$ for some $k=1, \ldots, n-1$, but then $y \mathcal{T}(\hat{P}) x$. This implies $y \mathcal{T}(P) x$, contradicting $x T y$. Therefore, $P^{\prime}$ is acyclic, but this contradicts maximality of $\hat{P}$. Therefore, $y \in U T$.

For the converse direction, we use the following lemma, which is proved in the appendix. According to the lemma, there is an acyclic subrelation of $P$ emanating from an arbitrary $x$ such that every alternative "reachable" from $x$ under $P$ is also reachable from $x$ under the subrelation.

Lemma 1 For each $x \in X$, there exists an acyclic subrelation $\tilde{P} \subseteq P$ on $\mathcal{T}(P)^{-1}(x) \cup\{x\}$ such that $\mathcal{T}(\tilde{P})^{-1}(x)=\mathcal{T}(P)^{-1}(x) \backslash\{x\}$.

Take any $x \in U T$. Let $\tilde{P}$ be as in Lemma 1 . To see that $x$ is $\tilde{P}$ maximal in $Y=\mathcal{T}(P)^{-1}(x) \cup\{x\}$, suppose there exists $y \in Y$ such that $y \tilde{P} x$. Since $Y \backslash\{x\}=\mathcal{T}(\tilde{P})^{-1}(x)$, however, we then have $x \mathcal{T}(\tilde{P}) y$, contradicting acyclicity of $\tilde{P}$. Let $\hat{\mathbf{P}}$ be the set of acyclic subrelations $Q$ such that $\tilde{P} \subseteq$ $Q \subseteq P$. Let $\mathbf{Q}$ be a chain in $\hat{\mathbf{P}}$, and let $\hat{Q}=\bigcup \mathbf{Q}$. Clearly, $\tilde{P} \subseteq \hat{Q} \subseteq \bar{P}$. Since the class of acyclic relations is closed upward (see Duggan, 1999), $\hat{Q}$ is acyclic. Therefore, by Zorn's lemma, $\hat{\mathbf{P}}$ has an element, say $\hat{P}$, that is maximal with respect to set-inclusion. I claim that $x$ is maximal with respect to $\hat{P}$, i.e., $x \in M(\hat{P})$. Otherwise, there exists $y \in X$ such that y $\hat{P} x$, which implies $y P x$. Since $x \in U T$, we must then have $x \mathcal{T}(P) y$, implying $y \in Y \backslash\{x\}=\mathcal{T}(\tilde{P})^{-1}(x)$. Therefore, we have $x \mathcal{T}(\tilde{P}) y$, which implies $x \mathcal{T}(\hat{P}) y$, contradicting acyclicity of $\hat{P}$. Therefore, $x \in M(\hat{P})$, which completes the proof of the theorem.

From Theorems 3 and 4, we have the following immediate consequence for the location of acyclic choices: every alternative in the strong top cycle is chosen by some maximal acyclic subrelation of $P$, and all choices generated in this manner must lie in the weak top cycle set. In case $P$ is total, these sets coincide, yielding a new interpretation of the familiar top cycle in terms of acyclic choice. 


\section{Corollary 2}

$$
S T C \subseteq \bigcup_{\hat{P} \in \mathbf{P}_{A}^{*}} M(\hat{P}) \subseteq W T C
$$

If $P$ is total, then

$$
T C=\bigcup_{\hat{P} \in \mathbf{P}_{A}^{*}} M(\hat{P})
$$

The characterization in Theorem 4 raises interest in the connections between the untrapped set and other solutions. In general, it can be seen that $U X C$ and $U T$ are not logically nested, and in fact either can be a proper subset of the other. For one inclusion, let $X=\{a, b, c\}$, and let $P=\{(a, b),(b, c)\}$; then $U X C=X$, while $U T=\{a\}$. For the other, the second example following Theorem 1 shows that the extended uncovered set can be a proper subset of the top cycle set. Since $P$ is total in that example, the top cycle coincides with the untrapped set, as needed.

Theorem 3 establishes that the untrapped set is nested between the top cycle sets. In this respect, the untrapped set is similar to the "mixed saddle," defined by Duggan and Le Breton $(1997,1999,2001)$ as a noncooperative game-theoretic solution to a particular two-player, symmetric, zero-sum game derived from the relation $P$. Let the pure strategy sets of the players be $X$, which we now assume finite, and let $\Pi$ be the payoff function for player 1 defined by

$$
\Pi(x, y)=\left\{\begin{array}{cl}
1 & \text { if } x P y \\
-1 & \text { if } y P x \\
0 & \text { else. }
\end{array}\right.
$$

Let $\sigma$ and $\sigma^{\prime}$ denote probability distributions on $X$, representing mixed strategies, and let $\Pi\left(\sigma, \sigma^{\prime}\right)$ denote the expected payoff to player 1 from $\sigma$, when player 2 uses strategy $\sigma^{\prime}$. For a mixed strategy degenerate on one alternative, we simply write that alternative in the argument of $\Pi$.

A subset $Y \subseteq X$ is a mixed generalized saddle point (MGSP) if, for all $x \in X \backslash Y$, there exists a distribution $\sigma$ with support in $Y$ such that, for all $y \in Y, \Pi(\sigma, y)>\Pi(x, y)$. That is, $Y$ is a MGSP if every alternative outside the set is strictly dominated by a mixed strategy, if we vary player 2's strategies within the set $Y$. We say the MGSP $Y$ is a mixed saddle if 
it is minimal among MGSP's with respect to set-inclusion. Duggan and Le Breton $(1997,1999,2001)$ show that there is a unique mixed saddle, which we denote $M S$. These papers establish that the mixed saddle, like the untrapped set, is nested between the top cycle sets:

$$
S T C \subseteq M S \subseteq W T C .
$$

Moreover, Duggan and Le Breton show that the mixed saddle coincides with the unique minimal "rationalizable set," meaning that it contains all best responses to mixed strategies with support within the mixed saddle, and it is the unique minimal set with this property.

The following theorem establishes the relationship between the untrapped set and the mixed saddle: the untrapped set offers a refinement of the mixed saddle.

Theorem 5 Assume $X$ is finite. Then $U T \subseteq M S$.

To prove the theorem, take any $x \in U T$. To see that $x \in M S$, suppose otherwise. Let $\sigma$ be any mixed strategy equilibrium. Duggan and Le Breton (1999) show that $\sigma(M S)=1$, and by symmetry it follows that $\Pi(\sigma, \sigma)=0$. If $\Pi(x, \sigma)=0$, then $x$ is a best response to $\sigma$, and since $M S$ is a rationalizable set, it follows that $x \in M S$. If $\Pi(x, \sigma)<0$, then there exists some $y \in X$ with $\sigma(y)>0$ such that $y P x$. Note that $y \in M S$. Since $x$ is untrapped, we have $x \mathcal{T}(P) y$, i.e., there exist a natural number $n$ and alternatives $x_{1}, \ldots, x_{n} \in X$ such that

$$
x=x_{1} P x_{2} \cdots x_{n-1} P x_{n}=y .
$$

Note that $\Pi\left(x_{n-1}, y\right)=1$, so $x_{n-1}$ is a best response to $y$. Since $y \in M S$ and $M S$ is a rationalizable set, we have $x_{n-1} \in M S$. Similarly, $x_{n-2} \in$ $M S$, and an induction argument based on this logic yields $x=x_{1} \in M S$, a contradiction. Therefore, $x \in M S$, which completes the proof of the theorem.

To see that the inclusion stated in Theorem 5 may be strict, consider the following weak tournament. Let $X=\{a, b, c, d, e\}$, and let $P=\{(b, c),(c, d)$, $(d, e),(e, c)\}$. Note that $b$ traps $c$, so that $c \notin U T$. However, $c \in M S$, for $a \in M S$ because $a \in M(P) ; c$ is a best response to $a$; and since $M S$ is a rationalizable set, we must have $c \in M S$. 


\section{Choice via Negative Transitivity: The Weak Top Cycle}

We have considered the choices generated by maximal transitive subrelations, and then we considered the choices generated by the weaker condition of acyclicity. In this section, we consider subrelations that satisfy negative transitivity, a more demanding condition than transitivity that entails that the alternatives can be ranked. ${ }^{7}$ Let

$$
\mathbf{P}_{N}=\{\hat{P} \subseteq P \mid \hat{P} \text { is negatively transitive }\}
$$

denote the negatively transitive subrelations of $P$, and let $\mathbf{P}_{N}^{*}$ denote the elements of $\mathbf{P}_{N}$ that are maximal with respect to set-inclusion. The next result establishes that the choices generated by maximal negatively transitive subrelations coincide with the weak top cycle. Note the departure from the structure of the above results, most notably in that coincidence with the weak top cycle holds for all maximal negatively transitive subrelations: taking unions is not needed.

Theorem 6 Let $\hat{P} \in \mathbf{P}_{N}$ be any negatively transitive subrelation. Then

$$
W T C \subseteq M(\hat{P}) .
$$

If $\hat{P} \in \mathbf{P}_{N}^{*}$ is a maximal negatively transitive subrelation, then

$$
W T C=M(\hat{P}) .
$$

To prove the theorem, let $\hat{P} \in \mathbf{P}_{N}$, and take any $x \in W T C$. If $x \notin$ $M(\hat{P})$, then there is some $y \in X$ such that $y \hat{P} x$. Since $x \in W T C$, we have $x \mathcal{T}(R) y$, so there exist a natural number $n$ and alternatives $x_{1}, \ldots, x_{n} \in X$ such that

$$
x=x_{1} R x_{2} \cdots x_{n-1} R x_{n}=y .
$$

By negative transitivity, $y \hat{P} x$ implies that either $y \hat{P} x_{n-1}$ or $x_{n-1} \hat{P} x$. The former case, however, implies $x_{n}=y P x_{n-1}$, which does not hold. Therefore, $x_{n-1} \hat{P} x$. Similarly, we have $x_{n-2} \hat{P} x$, and an induction argument based on this logic yields $x_{2} \hat{P} x=x_{1}$. But this implies $x_{2} P x_{1}$, a contradiction. Therefore, $x \in M(\hat{P})$.

\footnotetext{
${ }^{7}$ Given an asymmetric, negatively transitive subrelation $\hat{P}$ of $P$, the corresponding weak preference relation $\hat{R}$ is complete and transitive, and the corresponding indifference relation is transitive.
} 
Now let $\hat{P} \in \mathbf{P}_{N}^{*}$ be maximal, take any $y \in M(\hat{P})$, and suppose $y \notin$ $W T C$. Then there exists $x \in X$ such that $x \mathcal{T}(R)$ y and not $y \mathcal{T}(R) x$. Define $P^{\prime}$ as follows:

$$
P^{\prime}=\hat{P} \cup\{(z, w) \mid z \in \mathcal{T}(R)(x), w \notin \mathcal{T}(R)(x)\} .
$$

Clearly, $(x, y) \in P^{\prime}$. And given $z \in \mathcal{T}(R)(x)$ and $w \notin \mathcal{T}(R)(x)$, we must have $z P w$, so $P^{\prime} \subseteq P$. To see that $P^{\prime}$ is negatively transitive, take any $s, t \in X$ such that $s P^{\prime} t$, and take any $u \in X$. If $(s, t) \in \hat{P}$, then, by negative transitivity of $\hat{P}$, we have $x \hat{P} u$ or $u \hat{P} t$. This implies $x P^{\prime} u$ or $u P^{\prime} t$, as required. If $(s, t) \in P^{\prime} \backslash \hat{P}$, then $s \in \mathcal{T}(R)(x)$ and $t \notin \mathcal{T}(R)(x)$, and there are two possibilities: $u \in \mathcal{T}(R)(x)$ and $u \notin \mathcal{T}(R)(x)$. In the former case, we have $u P^{\prime} t$ by construction; in the latter, we have $s P^{\prime} u$, as required. Therefore, $P^{\prime}$ is negatively transitive, contradicting maximality of $\hat{P}$. Therefore, $y \in W T C$, which completes the proof of the theorem.

\section{Other Procedures}

We have considered the choices generated by subrelations belonging to several classes isolated by the standard rationality properties of acyclicity, transitivity, and negative transitivity. I end by considering the choices generated by two polar classes of subrelations that, in a sense, bound the properties analyzed above. We will see that these two routes to non-empty choice sets fail to yield useful results. I first consider the choices generated by maximal subrelations possessing maximal elements - in the finite setting, this weakens the requirement of acyclicity. Let

$$
\mathbf{P}_{K}=\{\hat{P} \subseteq P \mid M(\hat{P}) \neq \emptyset\}
$$

denote the subrelations of $P$ possessing maximal elements, and let $\mathbf{P}_{K}^{*}$ denote the elements of $\mathbf{P}_{K}$ that are maximal with respect to set inclusion. Though it may seem that the difference between acyclicity and non-empty maximal sets is slight, the next proposition establishes that the latter approach is no help: unless a $P$-maximal alternative already exists, this choice procedure fails to reject any alternative.

Proposition 3 If $M(P)=\emptyset$, then

$$
X=\bigcup_{\hat{P} \in \mathbf{P}_{K}^{*}} M(\hat{P}) .
$$


The proof is trivial. Take any alternative $x$, and define $\hat{P}$ by removing all strict preferences over $x$, i.e.,

$$
\hat{P}=\{(z, w) \in P \mid w \neq x\} .
$$

Clearly, $x$ is $\hat{P}$-maximal. Assuming $M(P)=\emptyset$, we actually have $M(\hat{P})=$ $\{x\}$. Thus, $\hat{P}$ is maximal with respect to set-inclusion in $\mathbf{P}_{K}$, as required.

At the polar extreme, we might consider strengthening negative transitivity by adding the requirement that the subrelation be total. In other words, we might consider the choices generated by maximal elements of all "linear order" subrelations of $P$. But it is clear that $P$ possesses a linear subrelation if and only if $P$ itself is a linear order, in which case the only such subrelation is $P$ itself. In this case, the existence of a $P$-maximal alternative reduces to the existence of an alternative ranked atop the linear ordering $P$, and, in the absence of such an alternative, the attempt to go to a linear subrelation fails to solve the non-existence problem.

A related approach is to consider subrelations that are linear but only on their domain. Such relations are "chains," and the approach would be to unify the maximal elements of maximal chains. This is proposed by Banks (1985) and produces what is known as the Banks set. In finite settings, this yields a subset of the uncovered set with a close connection to sophisticated voting in agendas: the Banks set consists of all outcomes of sophisticated voting in amendment agendas for all orders of alternatives. A distinction of the procedures I have considered, which are based on more conventional rationality conditions, is that the choices produced by them have simple binary representations: for example, the alternatives chosen by a maximal transitive subrelation are just those that are not covered. Due to the structure of the class of maximal chains, such a simple binary representation does not appear to be available for the Banks set.

\section{A Proof of Lemma}

Lemma 1 For each $x \in X$, there exists an acyclic subrelation $\tilde{P} \subseteq P$ on $\mathcal{T}(P)^{-1}(x) \cup\{x\}$ such that $\mathcal{T}(\tilde{P})^{-1}(x)=\mathcal{T}(P)^{-1}(x) \backslash\{x\}$.

Let $Y=\mathcal{T}(P)^{-1}(x) \cup\{x\}$. Let $\tilde{\mathbf{P}}$ denote the set of acyclic subrelations $\tilde{P} \subseteq P$ such that $\tilde{P} \subseteq Y \times Y$ and, for all $y, z \in Y$, if $y \tilde{P} z$, then $x=y$ 
or $x \mathcal{T}(\tilde{P}) y$. Note that $x$ is necessarily $\tilde{P}$-maximal in $Y$. Since the empty relation lies in $\tilde{\mathbf{P}}$, this set is non-empty. Let $\mathbf{Q}$ be a chain in $\tilde{\mathbf{P}}$, and let $\bar{Q}=\bigcup \mathbf{Q}$. Clearly, $\bar{Q} \subseteq P \cap(Y \times Y)$, and since the class of acyclic relations is closed upward, $\bar{Q}$ is acyclic. Take any $y, z \in Y$ such that $y \bar{Q} z$. Thus, there exists $Q \in \mathbf{Q}$ such that $y Q z$, and therefore $x=y$ or $x \mathcal{T}(Q) y$, which implies $x=y$ or $x \mathcal{T}(\bar{Q}) y$. Therefore, $\bar{Q} \in \tilde{\mathbf{P}}$. By Zorn's lemma $\tilde{\mathbf{P}}$ possesses an element, say $\tilde{P}$, that is maximal with respect to set-inclusion. To show that $\mathcal{T}(\tilde{P})^{-1}(x)=Y \backslash\{x\}$, suppose there exists $y \in(Y \backslash\{x\}) \backslash \mathcal{T}(\tilde{P})^{-1}(x)$. Since $y \in Y$, there exist a natural number $n$ and alternatives $x_{1}, \ldots, x_{n} \in Y$ such that

$$
x=x_{1} P x_{2} \cdots x_{n-1} P x_{n}=y,
$$

and, since $x \neq y$, we may assume that the elements $x_{1}, \ldots, x_{n}$ are distinct. Now define

$$
P^{\prime}=\tilde{P} \cup\left\{\left(x_{1}, x_{2}\right), \ldots,\left(x_{n-1}, x_{n}\right)\right\} .
$$

Clearly, $P^{\prime} \subseteq P \cap(Y \times Y)$. Furthermore, for all $w, z \in Y$, if $w P^{\prime} z$, then $x=w$ or $x \mathcal{T}\left(P^{\prime}\right) w$. Therefore, by maximality of $\tilde{P}$, it must be that $P^{\prime}$ contains a cycle, so there exists $s \in Y$ such that $s \mathcal{T}\left(P^{\prime}\right) s$. Thus, there exist a natural number $m$ and alternatives $z_{1}, \ldots, z_{m} \in Y$ such that

$$
s=z_{0} P^{\prime} z_{1} \cdots z_{m-1} P^{\prime} z_{m}=s .
$$

Since $\tilde{P}$ is acyclic, there must exist $k=1, \ldots, n-1$ and $\ell=0,1, \ldots, m-1$ such that $\left(x_{k}, x_{k+1}\right)=\left(z_{\ell}, z_{\ell+1}\right)$. Consider the largest $k$ for which there exist such $s, m, z_{0}, \ldots, z_{m}$, and $\ell$. It follows that there is no $j=1, \ldots, n-1$ such that $\left(z_{\ell+1}, z_{\bmod (\ell+2, m)}\right)=\left(x_{j}, x_{j+1}\right)$, for suppose otherwise: since $x_{k}=z_{\ell} P$ $z_{\ell+1}=x_{j}$, we cannot have $j=k$; and so $j<k$, but then $x_{k+1}=z_{\ell+1}=x_{j}$ contradicts distinctness of $x_{1}, \ldots, x_{n}$. Thus, from $z_{\ell+1} P^{\prime} z_{\bmod (\ell+2, m)}$, we have $z_{\ell+1} \tilde{P} z_{\bmod (\ell+2, m)}$. Since $\tilde{P} \in \tilde{\mathbf{P}}$, we have $x=z_{\ell+1}$ or $x \mathcal{T}(\tilde{P}) z_{\ell+1}$. As $x$ is $\tilde{P}$-maximal in $Y$, this leaves the latter case. Using $z_{\ell+1}=x_{k+1}$, we have

$$
x \mathcal{T}(\tilde{P}) x_{k+1} P x_{k+2} \cdots x_{n-1} P x_{n}=y .
$$

Now define

$$
P^{\prime \prime}=\tilde{P} \cup\left\{\left(x_{k+1}, x_{k+2}\right), \ldots,\left(x_{n-1}, x_{n}\right)\right\},
$$

so that $x \mathcal{T}\left(P^{\prime \prime}\right) y$. Clearly, $P^{\prime \prime} \subseteq P \cap(Y \times Y)$. Moreover, $P^{\prime \prime}$ is acyclic, by choice of $k$. And for all $w, z \in Y$, if $w P^{\prime \prime} z$, then $x=w$ or $x \mathcal{T}\left(P^{\prime \prime}\right) w$. Therefore, $P^{\prime \prime} \in \tilde{\mathbf{P}}$, contradicting maximality of $\tilde{P}$. Therefore, we must have $\mathcal{T}(\tilde{P})^{-1}(x)=Y \backslash\{x\}$, completing the proof. 


\section{References}

[1] D. Austen-Smith and J. Banks (1999) Positive Political Theory I: Collective Preference, University of Michigan Press: Ann Arbor.

[2] J. Banks (1985) "Sophisticated Voting outcomes and Agenda Control," Social Choice and Welfare, 1: 295-306.

[3] J. Banks (1995) "Acyclic Social Choice from Finite Sets," Social Choice and Welfare, 12: 293-310.

[4] J. Banks, J. Duggan, and M. Le Breton (2002) "Bounds for Mixed Strategy Equilibria in the Spatial Model of Elections," Journal of Economic Theory, 103: 88-105.

[5] J. Banks, J. Duggan, and M. Le Breton (2003) "Social Choice and Electoral Competition in the General Spatial Model," mimeo.

[6] J. Duggan (1999) "A General Extension Theorem for Binary Relations," Journal of Economic Theory, 86: 1-16.

[7] J. Duggan (2004) "Uncovered Sets," mimeo.

[8] J. Duggan and M. Le Breton (1997) "Dominance-based Solutions for Strategic Form Games," mimeo.

[9] J. Duggan and M. Le Breton (1999) "Mixed Refinements of Shapley's Saddles and Weak Tournaments," CORE Discussion Paper, no. 9921.

[10] J. Duggan and M. Le Breton (2001) "Mixed Refinements of Shapley's Saddles and Weak Tournaments," Social Choice and Welfare, 18: 65-78.

[11] B. Dutta and J.-F. Laslier (1999) Comparison Functions and Choice Correspondences, Social Choice and Welfare, 16: 513-532.

[12] P. Fishburn (1977) "Condorcet Social Choice Functions," SIAM Journal of Applied Mathematics, 33: 469-489.

[13] A. Mas-Colell and H. Sonnenschein (1972) "General Possibility Theorems for Group Decisions," Review of Economic Studies, 39: 185-192.

[14] R. McKelvey (1986) "Covering, Dominance, and Institution-free Properties of Social Choice, American Journal of Political Science, 30: 283314. 
[15] N. Miller (1980) "A New Solution Set for Tournaments and Majority Voting: Further Graph-theoretical Approaches to the Theory of Voting," American Journal of Political Science, 24: 68-96.

[16] H. Moulin (1986), "Choosing from a Tournament," Social Choice and Welfare, 3: 271-291.

[17] K. Nakamura (1979) "The Vetoers in a Simiple Game with Ordinal Preferences," International Journal of Game Theory, 8: 55-61.

[18] T. Schwartz (1986) The Logic of Collective Action, Columbia University Press: New York.

[19] T. Schwartz (2001) "From Arrow to Cycles, Instability, and Chaos by Untying Alternatives," Social Choice and Welfare, 18: 1-22.

[20] A. Sen (1970) "The Impossibility of a Paretian Liberal," Journal of Political Economy, 78: 152-157.

[21] M. Walker (1977) "On the Existence of Maximal Elements," Journal of Economic Theory, 16: 470-474. 